\title{
Pure Robotic Retrocaval Ureter Repair
}

\author{
Ashok K. Hemal, Ranjit Rao, Sachit Sharma, Rhys G. E. Clement \\ Department of Urology (AKH), Wake Forest University Health Sciences, Winston-Salem, North \\ Carolina, USA and Department of Urology (RR, SS, RGEC), All India Institute of Medical Sciences, \\ New Delhi, India
}

\begin{abstract}
Purpose: To demonstrate the feasibility of pure robotic retrocaval ureter repair.

Materials and Methods: A 33 year old female presented with right loin pain and obstruction on intravenous urography with the classical "fish-hook" appearance. She was counseled on the various methods of repair and elected to have a robot assisted repair. The following steps are performed during a pure robotic retrocaval ureter repair. The patient is placed in a modified flank position, pneumoperitoneum created and ports inserted. The colon is mobilized to expose the retroperitoneal structures: inferior vena cava, right gonadal vein, right ureter, and duodenum. The renal pelvis and ureter are mobilized and the renal pelvis transected. The ureter is transposed anterior to the inferior vena cava and a pyelopyelostomy is performed over a JJ stent.

Results: This patient was discharged on postoperative day 3. The catheter and drain tube were removed on day 1 . Her JJ stent was removed at 6 weeks postoperatively. The postoperative intravenous urography at 3 months confirmed normal drainage of contrast medium.

Conclusion: Pure robotic retrocaval ureter is a feasible procedure; however, there does not appear to be any great advantage over pure laparoscopy, apart from the ergonomic ease for the surgeon as well the simpler intracorporeal suturing.
\end{abstract}

Key words: ureter; vena cava; abnormalities; laparoscopy; robotics

Int Braz J Urol. 2008; 34: 734-8

\section{INTRODUCTION}

Retrocaval ureter is an unusual urological problem that may require operative repair (Figure-1). The first case of retrocaval ureter repair was published in 1949 by Anderson and Hynes (1). The classical approach is an open technique of transposing the ureter anteriorly to the inferior vena cava followed by ureteroureterostomy. Laparoscopic retrocaval ureter repairs have also been performed but can be technically challenging. This is the first case of a pure robotic repair, to our knowledge, performed in an adult. We present our robotic technique of pure robotic retrocaval ureter repair.

\section{SURGICAL TECHNIQUE}

1. Patient position - The patient is positioned in a modified flank position over the kidney break at a 45 degree angle. The patient is then adequately secured with supports and strapping, and all pressure areas are protected.

2. Port position - A Veres needle is used to create a pneumoperitoneum, then a $10 \mathrm{~mm}$ port is inserted for the camera at the level of the umbilicus just lateral to the rectus abdominis muscle. Two $8 \mathrm{~mm}$ ports are inserted for the robotic arms, one under the costal margin in the midclavicular line and the other at two thirds of the way along McBurney's line (anterior 


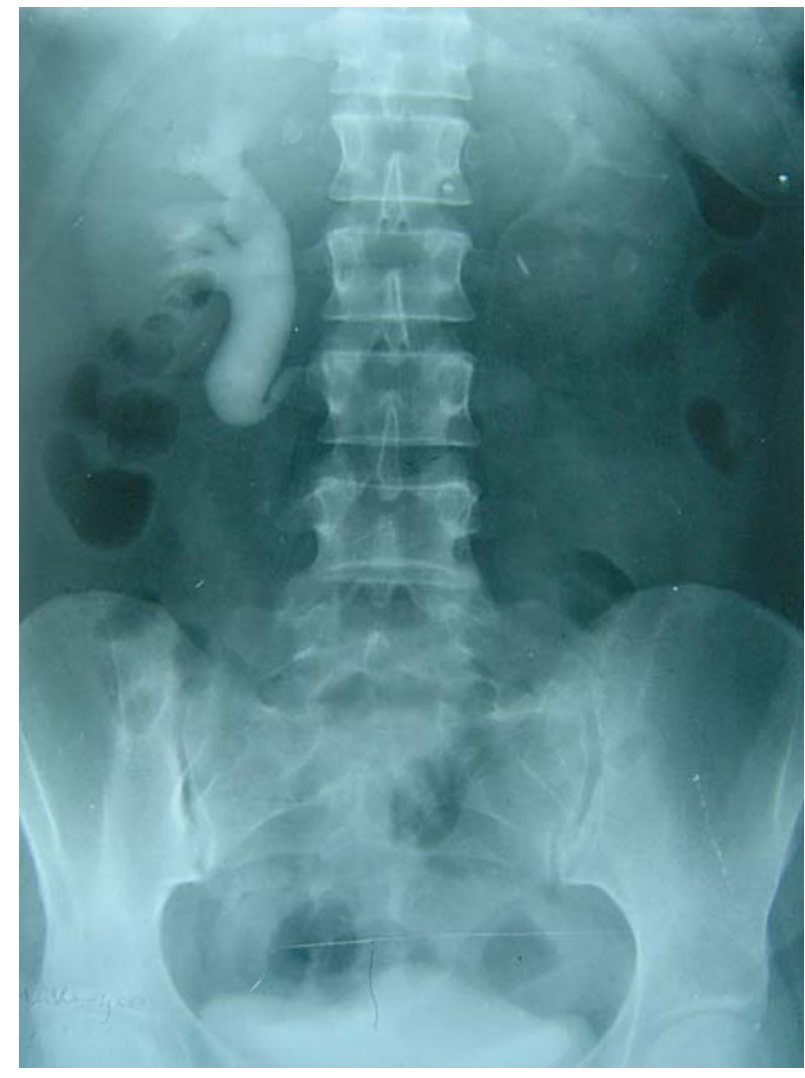

Figure 1-Pre-operative IVU.

superior iliac spine and umbilicus). A further $5 \mathrm{~mm}$ port is inserted $3 \mathrm{~cm}$ below the camera port for the assistant to perform retraction and suction. The robot is then docked. The whole process of pneumoperitoneum, port insertion and docking takes 15 minutes.

3. Colon mobilization - The hepatic flexure and right colon are mobilized medially to provide exposure to the right retroperitoneal structures.

4. Exposure of retroperitoneal structures: (Figure-2). The right renal pelvis, inferior vena cava, right gonadal vein, right ureter and duodenum are all identified.

5. Mobilization of renal pelvis and ureter: (Figure-3). The right renal pelvis is dissected free from its surrounding fascial layers. The proximal right ureter is dissected free where it can be seen to disappear superiorly under the inferior vena cava.

6. Transection of ureteropelvic junction: (Figure-4). The renal pelvis is transected and the

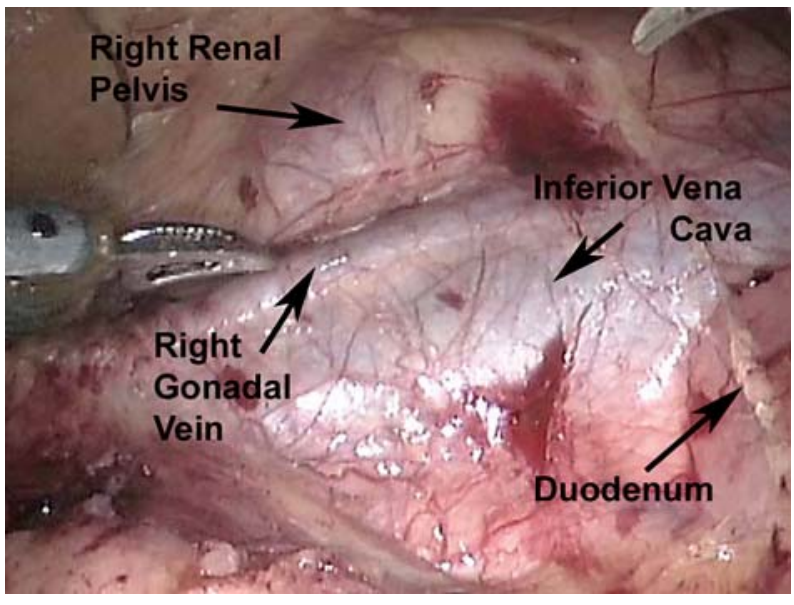

Figure 2-Exposure.

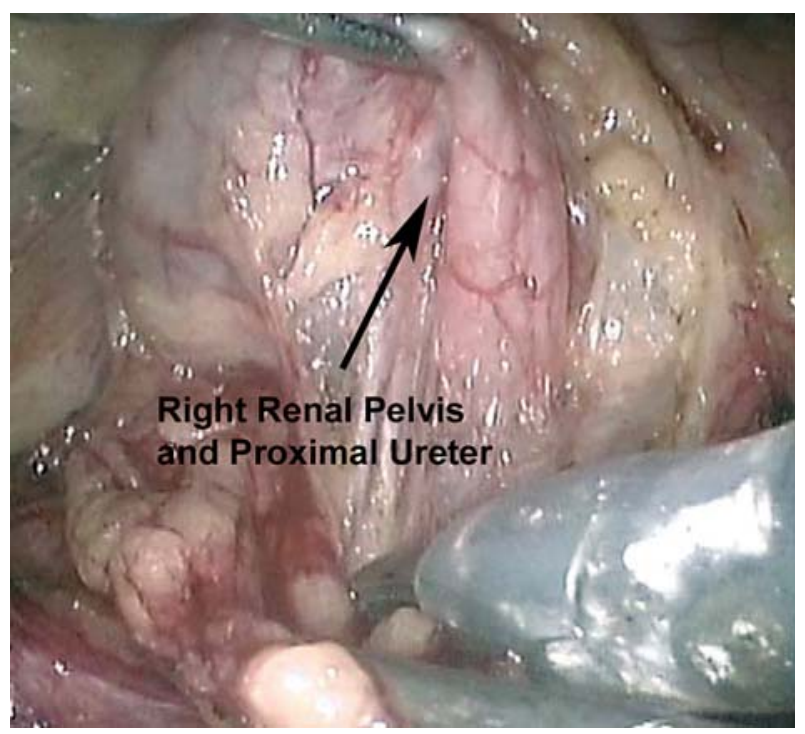

Figure 3 - Mobilization of renal pelvis and ureter.

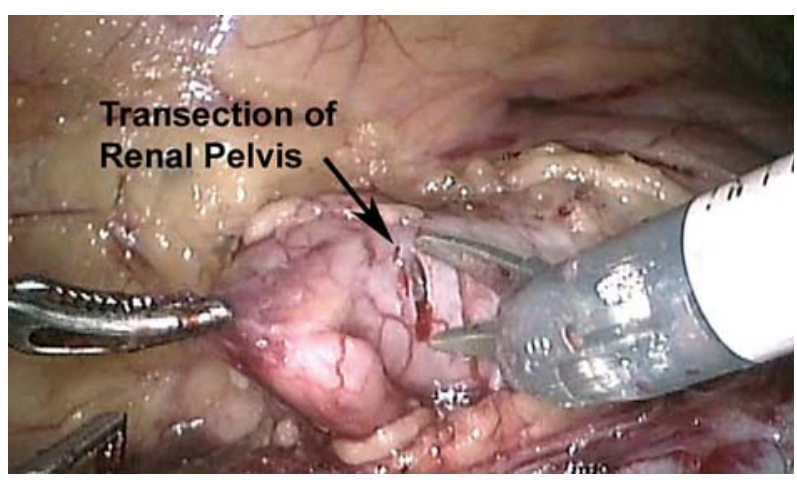

Figure 4-Transection of renal pelvis. 


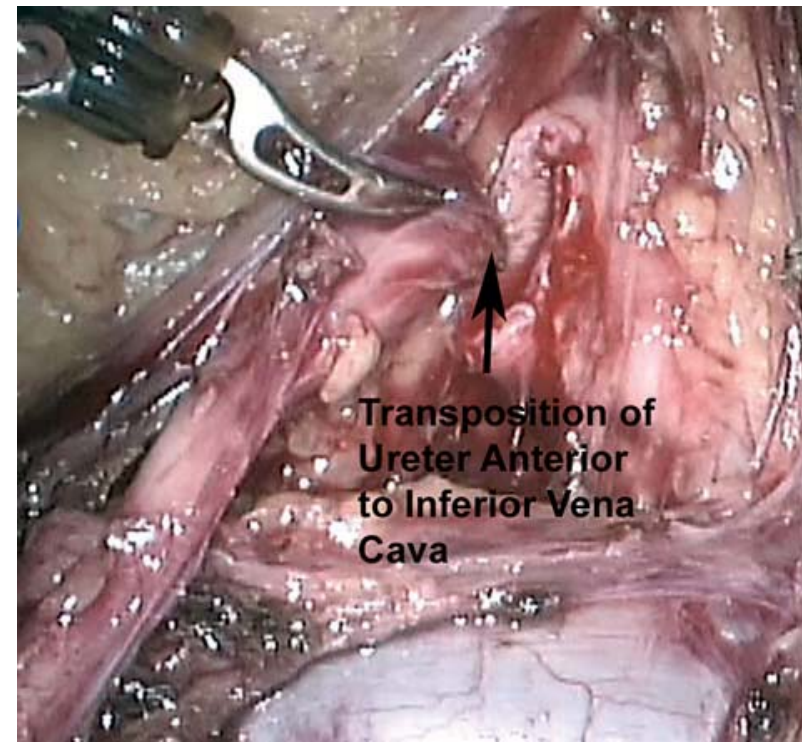

Figure 5-Transposition of ureter.

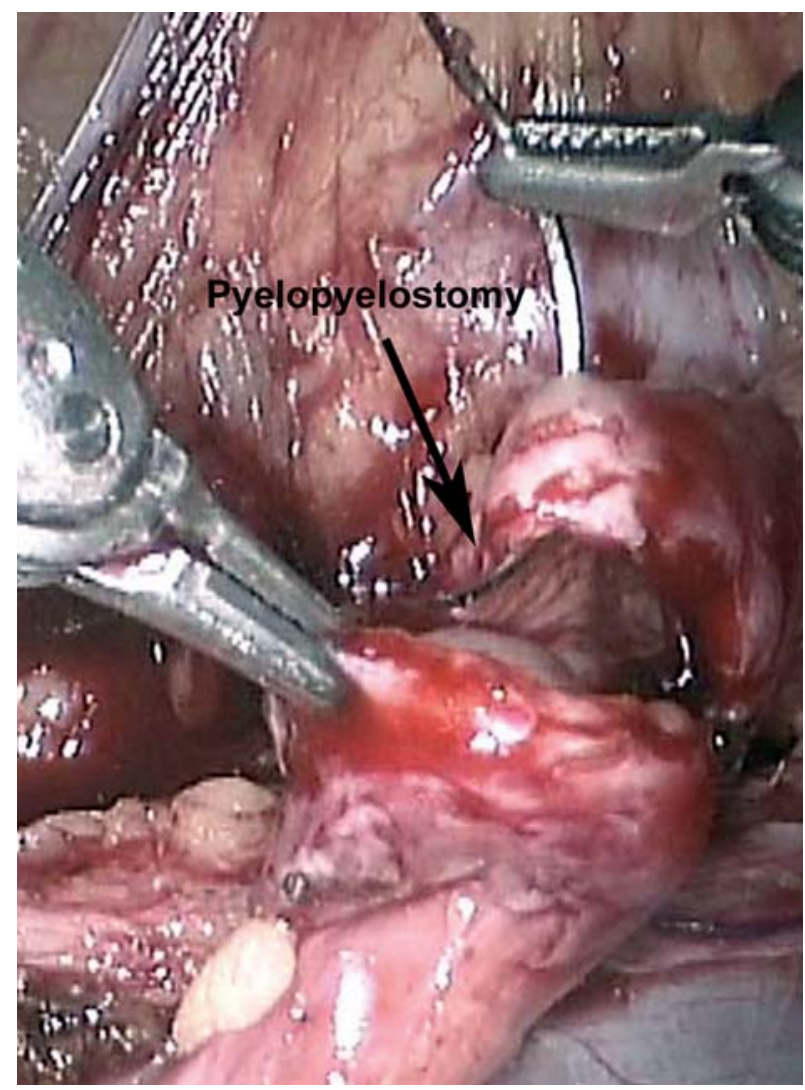

Figure 6-Pyelopyelostomy. ureteropelvic junction along with the retrocaval segment are transposed anterior to the inferior vena cava (Figure-5) in preparation for a pyelopyelostomy. This may not be possible for lower segment retrocaval ureters in which case ureteroureterostomy must be performed.

7. Pyelopyelostomy: (Figure-6). Performing a pyelopyelostomy is easier than a ureteroureterostomy and one is less likely to produce stricture formation due to the larger caliber structures as well as the better blood supply as one goes more superiorly. This is performed with 40 polygalactin suture material in an interrupted fashion.

8. Antegrade JJ stent insertion: (Figure-7). Prior to closing the anastomosis, a $6 \mathrm{~F} \mathrm{JJ}$ stent is inserted in an antegrade fashion. The stent with the wire is introduced via the $5 \mathrm{~mm}$ port. It is grasped using the robotic needle holder, introduced into the ureter and passed down to the bladder.

9. Drain tube insertion: The robot is undocked and a drain tube is inserted via the $5-\mathrm{mm}$ port. The 10 $\mathrm{mm}$ port is closed in standard fashion and an indwelling catheter is left in situ.

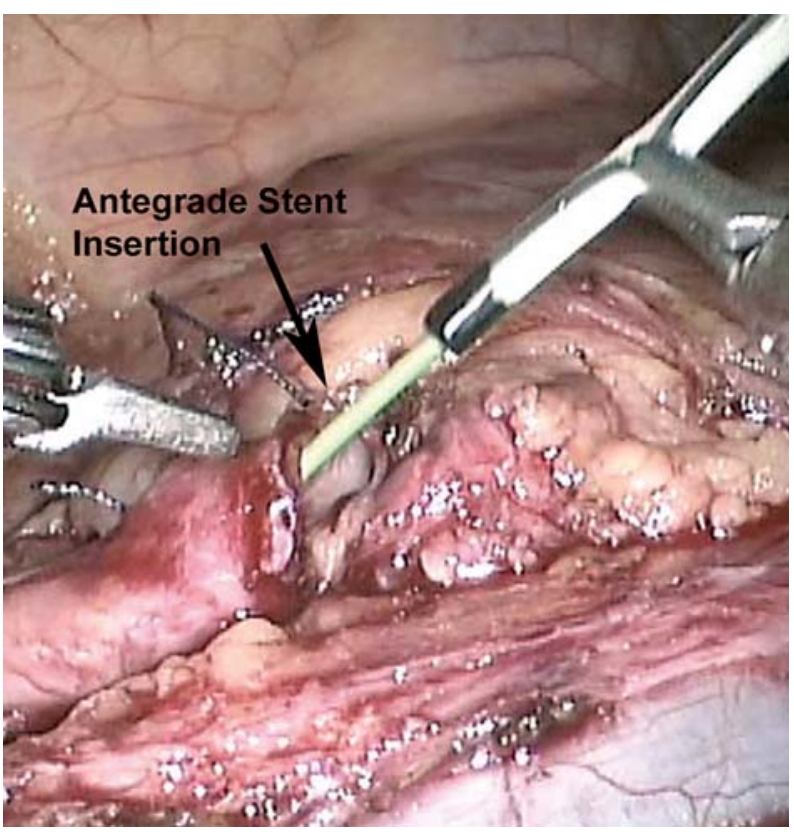

Figure 7 - Antegrade JJ stent insertion. 


\section{RESULTS}

This 33 year old female patient was discharged on postoperative day 3 . The catheter and drain tube were removed on day 1 . The JJ stent was removed at 6 weeks post operatively. The post operative IVU at 3 months confirmed normal drainage of contrast (Figure-8).

\section{COMMENTS}

Robotic technology has become incorporated into certain areas of urology as in robotic prostatectomy and has become well accepted. Reconstructive urology represents a challenge for the robotic urologist to offer this technology safely, with efficacy over proven techniques and without increased morbidity.

Our case demonstrates the feasibility of a procedure using the robot but does not necessarily justify its use over other modalities. Though the fundamental surgical principles of a tension free, well vascularized anastamosis remain the same, patients may now receive the benefits of a minimally invasive approach, namely: smaller incision; better cosmetic effect, decreased pain; shorter hospital stay and a quicker return to normal activities. This holds true for both a pure laparoscopic or pure robotic approach.

Pure laparoscopic repair of the retrocaval ureter has been performed both transperitoneally and retroperitoneally (2). We have previously published our results with retroperitoneal ureterolysis and retrocaval ureter repair (3). Pure laparoscopic repair remains a technically challenging procedure, but in experts hands the results are excellent.

The robotic approach to retrocaval ureter was first published for a pediatric patient by Gundeti et al. in 2006 (4). Pyelopyelostomy with preservation of the retrocaval segment was first performed for a retrocaval ureter by Simfiroosh et al. in 2006 in a pure laparoscopic procedure (5). This preservation of the retrocaval segment does not appear to hinder drainage and it makes the anastamosis far easier to perform and may lead to a lower stricture rate.

The main advantage of the robotic technology is the ease of dissection and intracorporeal suturing. Expert laparoscopic surgeons may argue that there is

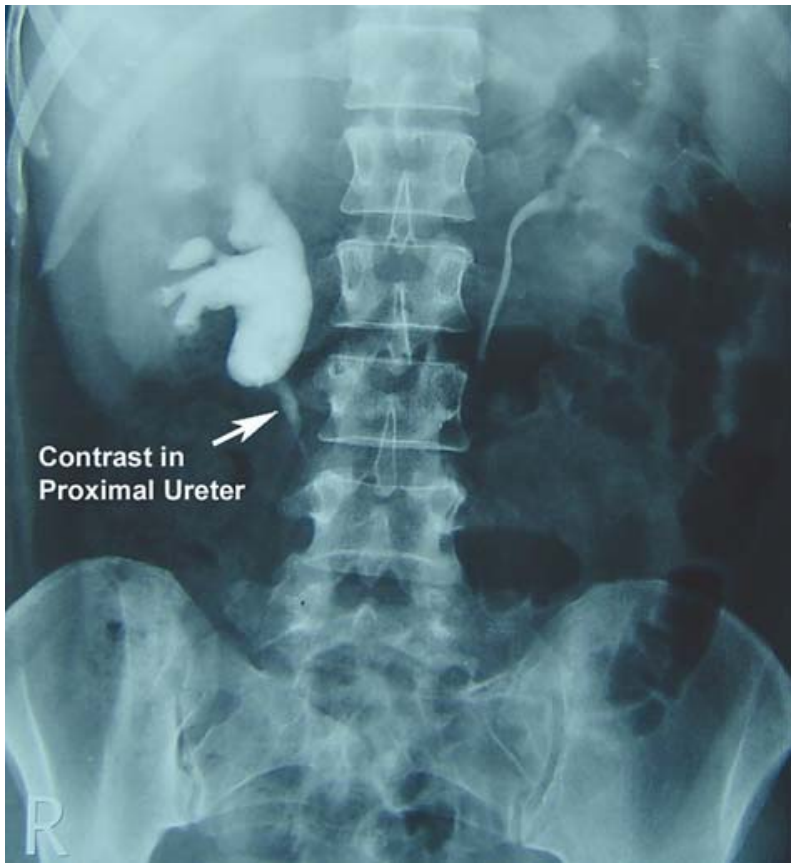

Figure 8-Postoperative IVU.

no need for the robot in such a procedure in the same way that laparoscopic pyeloplasty can be done without the robot. This of course is true, however the fact remains that new technologies emerge and it seems that robotic technology is here to stay. The downside to the robotic approach is of course the cost.

Since acquiring the da-Vinci-S robot in 2006 we have performed many reconstructive procedures such as megaureter repair and pyeloplasty with robotic assistance. This is the first retrocaval ureter repair that we have performed using the robot.

\section{CONCLUSION}

We demonstrated in this case that pure robotic retrocaval ureter repair is feasible. Apart from the ergonomic and technical benefits that the robotic approach gives the surgeon, there does not appear to be any other advantage over laparoscopy.

\section{CONFLICT OF INTEREST}

None declared. 


\section{Robotic Retrocaval Ureter Repair}

\section{REFERENCES}

1. Anderson JC, Hynes W: Retrocaval ureter; a case diagnosed pre-operatively and treated successfully by a plastic operation. Br J Urol. 1949; 21: 209-14.

2. Matsuda T, Yasumoto R, Tsujino T: Laparoscopic treatment of a retrocaval ureter. Eur Urol. 1996; 29: 115-118.

3. Gupta NP, Hemal AK, Singh I, Khaitan A: Retroperitoneoscopic ureterolysis and reconstruction of retrocaval ureter. J Endourol. 2001; 15: 291-3.

\section{Correspondence address:}

Dr. Ashok K Hemal

Department of Urology

Wake Forest University School of Medicine

Medical Center Boulevard

Winston-Salem, NC, 27157, USA

Fax: + 1336 716-5711

E-mail: ahemal@wfubmc.edu
4. Gundeti MS, Duffy PG, Mushtaq I: Robotic-assisted laparoscopic correction of pediatric retrocaval ureter. J Laparoendosc Adv Surg Tech A. 2006; 16: 422-4.

5. Simforoosh N, Nouri-Mahdavi K, Tabibi A: Laparoscopic pyelopyelostomy for retrocaval ureter without excision of the retrocaval segment: first report of 6 cases. J Urol. 2006; 175: 2166-9; discussion 2169.

Accepted after revision: August 28, 2008 